\title{
Erratum to: Model Representations of a Heat Stroke of a Massive Body with an Internal Cavity
}

\author{
E. M. Kartashov ${ }^{a, *}$ and E. V. Nenakhov ${ }^{b, * *}$ \\ ${ }^{a}$ MIREA-Russian Technological University (Lomonosov Institute of Fine Chemical Technology), Moscow, Russia \\ ${ }^{b}$ Moscow Aviation Institute (National Research University), Moscow, Russia \\ *e-mail: kartashov@mitht.ru \\ **e-mail: newnew94@mail.ru \\ Submitted January 13, 2022; accepted for publication January 13, 2022
}

DOI: $10.1134 / \mathrm{S} 2070048222320011$

The affiliations for the authors should read as follows:

${ }^{a}$ MIREA-Russian Technological University (Lomonosov Institute of Fine Chemical Technology), Moscow, Russia

${ }^{b}$ Moscow Aviation Institute (National Research University), Moscow, Russia

The original article can be found online at

https://doi.org/10.1134/S2070048221060089 